\title{
Serviços ao Cliente e Marketing de Relacionamento no Setor Hoteleiro de Porto Alegre
}

\author{
Luiz Antonio Slongo \\ Rafael Müssnich
}

\begin{abstract}
Resumo
Este estudo objetiva examinar as correlações existentes entre serviços ao cliente e Marketing de Relacionamento nas transações comerciais entre redes hoteleiras de Porto Alegre e seus clientes organizacionais. Para tanto, foi realizada a adaptação da escala proposta por Wilson e Vlosky (1997) para a mensuração de Marketing de Relacionamento, desenvolvida para a indústria da madeira e seus derivados nos Estados Unidos, bem como geração de indicadores para mensuração do nível de oferta de serviços ao cliente. Os constructos analisados foram: dependência do cliente; nível de comparação com hotéis alternativos; investimentos no relacionamento; troca de informações; confiança e comprometimento. Os resultados obtidos por meio de modelagem de equações estruturais sugerem índices adequados de ajustamento para a estrutura teórica proposta sobre o comportamento dos atributos de Marketing de Relacionamento e serviços ao cliente, apontando para a confirmação inicial das hipóteses de pesquisa. Em função destes resultados, algumas conclusões e implicações gerenciais são analisadas no final deste estudo.
\end{abstract}

Palavras-chave: marketing de relacionamento; serviço ao cliente.

\section{Abstract}

This study aims to analyze the correlations between customer service and relationship marketing in the commercial exchange between hotels and its organizational clients. The scale developed and applied by Wilson and Vlosky (1997) to measure relationship marketing in the United States context was adapted to the brazilian service sector, as well as indicators were generated to measure the customer service level. The results obtained by structural equation modeling show good indexes of fit to the proposed theoretical structure concerning the behavior of the attributes of relationship marketing and customer service. Based on these results, some conclusions and managerial implications are analyzed in the end of the study.

Key words: relationship marketing; customer service . 


\section{Apresentação do Tema de Pesquisa}

O mercado altamente competitivo, caracterizado por fenômenos como a globalização, rápidos avanços em telecomunicações, transportes e processamento de informações, e ampliação das opções de escolha dos consumidores e compradores industriais impõe crescentes desafios às empresas (WEBSTER, 1992). No esforço para criar vantagens competitivas sustentáveis no longo prazo, e que não sejam passíveis de imitação por parte dos concorrentes (ROWE; BARNES, 1998), as empresas passam por verdadeiras redefinições em suas cadeias de valores. Neste processo, encontram vantagens competitivas em atividades de relevância estratégica para potenciais de diferenciação (PORTER, 1986), entre as quais destacam-se a oferta de serviços ao cliente (INNIS; LA LONDE, 1994) e a gestão de relacionamentos entre os pólos comprador e vendedor (DICKSON, 1997; VARADARAJAN; JAYACHANDRAN, 1999; WEBSTER, 1997). A valorização dos relacionamentos como importante estratégia capaz de gerar vantagem competitiva culminou no surgimento de uma área particular de interesse dentro da disciplina de marketing, cunhada por Berry (1983) como Marketing de Relacionamento.

Os processos de troca transcendem as transações puras e simples. Sheth e Parvatiyar (2000) ressaltam a importância de se considerar o Marketing de Relacionamento como fenômeno que parte do pressuposto de uma interação de longo prazo, em que ambas as partes participam na busca do estabelecimento de vantagens competitivas sobre seus concorrentes (MOHR; SPEKMAN, 1994).

A gestão de relacionamentos surge como a melhor estratégia para lidar com a intangibilidade(BERRY, 1983, 1994), sendo os eventuais problemas no provimento do serviço contornados por meio da criação de vínculos duradouros entre fornecedores e clientes. Grönroos (2000, p. 97) afirma que "gerenciar serviços está no centro da construção e manutenção de relacionamentos, ainda que o Marketing de Relacionamento esteja baseado também em outros aspectos, como a construção de redes e o estabelecimento de acordos de alianças e parcerias estratégicas". Grönroos (1995, p. 183) cita que "na implementação de uma estratégia de serviços, uma abordagem do Marketing de Relacionamento e uma capacidade excelente de marketing interativo fazem-se essenciais”.

Este estudo tem seu foco neste tema, que une relações de troca duradouras no contexto business-to-business e oferta de serviços ao cliente como fontes estratégicas de obtenção e manutenção de vantagens competitivas sustentáveis 
no longo prazo. O objetivo é examinar as relações causais entre oferta de serviços ao cliente e os atributos indicadores de Marketing de Relacionamento nos processos de troca de duas redes hoteleiras e seus clientes organizacionais.

Na primeira parte deste artigo, apresenta-se uma fundamentação teórica dos principais conceitos pertinentes à pesquisa, envolvendo serviços, serviços ao cliente, contexto hoteleiro, Marketing de Relacionamento e a escala de Wilson e Vlosky (1997). A seguir, aborda-se o método empregado para a análise dos dados, bem como da pesquisa propriamente dita e seus resultados. Por fim, algumas conclusões e implicações gerenciais e acadêmicas serão traçadas.

\section{Fundamentação TeÓRICA}

\section{Serviços e Serviços ao Cliente}

Há alguns anos, a maior parte do valor agregado de um produto advinha dos processos de produção; com a queda dos custos de produção, devido à sistematização e automação, o valor passa a ter origem em outros atributos que somente os serviços podem criar (QUINN et al., 1998). Zeithaml (1982) e Berry e Parasuraman (1992) afirmam que é a sinergia do tangível com o intangível que forma o total de benefícios procurados pelos clientes, propiciando a criação de um produto diferenciado e de valor agregado, aos olhos dos clientes. Os serviços não são objetos, e não podem ser tratados como tais (SHOSTACK, 1978). As empresas, conseqüentemente, se concentram em identificar as atividades de serviços essenciais em que consigam superar seus concorrentes. Lovelock e Wright (2001, p. 90) definem a oferta de serviços como "todos os elementos em uma experiência de serviço destinados a propiciar valor para os clientes”.

Os serviços são a oferta essencial e indispensável. Pode-se listar algumas de suas características básicas, que justificam o maior envolvimento dos consumidores com empresas prestadoras de serviços, quais sejam (BERRY; PARASURAMAN, 1992; ZEITHAML et al., 1985; ZEITHAML; BITNER, 2000): relação com os clientes, perecibilidade, intangibilidade, inseparabilidade e customização.

Os serviços ao cliente, por sua vez, possuem as mesmas características dos serviços propriamente ditos; entretanto assumem dimensão diferenciada, ao serem considerados não mais a oferta essencial, mas a oferta complementar ou ampliada de serviços (GRÖNROOS, 1995; LEVY; WEITZ, 1992), devendo prover valores adicionais significativos à cadeia de suprimentos e eficiência em custos (INNIS; LA LONDE, 1994). Segundo Bowen et al. (1989), os serviços ao cliente que 
acompanham os produtos podem ser denominados produto ampliado ou produto aumentado, sendo fonte potencial de diferenciação do produto; mas, apesar de ir além da oferta essencial, não necessariamente diferenciam a empresa dos concorrentes. Para estes autores, os serviços ao cliente incluem tanto componentes técnicos quanto de relacionamento. Levitt (1990) tem a mesma idéia de produto ampliado, defendendo que, para a obtenção da diferenciação, não basta dar ao cliente o que ele espera, o que o autor chama de produto esperado, mas oferecer mais do que ele pensa que necessita ou do que se acostumou a esperar.

\section{Marketing de Relacionamento}

O relacionamento entre vendedores e compradores existe desde que os homens começaram a negociar bens e serviços, no processo inicial de trocas. Hoje, porém, estes relacionamentos adquirem caráter de gestão estratégica (WILSON, 1995), decorrente da realidade da competição global em grande número de indústrias, o que colocou o poder nas mãos dos clientes. Esta realidade faz com que as organizações busquem maior comprometimento de seus clientes e fornecedores (PARVATIYAR; SHETH, 1994).

A distinção de Macneil (1974; 1978) entre transações discretas, de duração geralmente curta e comunicação limitada e ausência de laços estreitos entre as partes, e trocas relacionais, com orientação de longo prazo e ancoradas em relações de cooperação, confiança e comprometimento, ao demarcar os dois extremos, traz mais clareza ao entendimento do Marketing de Relacionamento. Este processo de interação relacional remete ao conceito proposto por Morgan e Hunt (1994) de que Marketing de Relacionamento são todas as atividades de marketing direcionadas ao estabelecimento, desenvolvimento e manutenção de trocas relacionais bem sucedidas. Trocas relacionais bem sucedidas implicam ganhos mútuos com orientação de longo prazo, isto é, ganhos permanentes maximizados no longo prazo, o que pode ser aplicado a um amplo espectro de situações de troca de acordo com as peculiaridades de cada mercado.

A gestão de relacionamentos entre organizações compreende um processo organizado que desenvolve e analisa atitudes e ações, que culminam em relações duradouras com benefícios mútuos e objetivos comuns (JACKSON, 1985a, 1985b; MOHR; SPEKMAN, 1994; WEBSTER, 1984). Programas de gestão com orientação à construção de vínculos fortes e duradouros com clientes podem ser extremamente bem sucedidos em contextos apropriados. Em contrapartida, podem ser dispendiosos e pouco efetivos em terrenos onde há ênfase em vendas individuais (GANESAN, 1994; JACKSON, 1985a, 1985b). Existem empresas que não buscam relacionamentos duradouros como política de negócios, preferindo tratar cada transação como negociação diferente (MACNEIL, 1974; JACKSON, 1985a, 
1985b; DWYER et al., 1987). Neste sentido, Jackson (1985a, 1985b) apresenta um continuum classificatório de modelos de comportamento de compradores no ambiente organizacional, denominando as extremidades de always-a-share relações de curto prazo, com custos reduzidos de mudança e sem investimentos em ativos específicos para a transação - e lost-for-good - relações de longo prazo, com interações contínuas entre as partes ao longo do tempo, objetivos partilhados e busca por benefícios mútuos.

Sheth e Parvatiyar (2000), em concordância com a classificação das tendências de pesquisa de Wilson (1995), apontam as questões de identificação, definição e mensuração de constructos destinados à avaliação do desempenho dos relacionamentos como prioridade de pesquisa no nível conceitual. Esta direção é confirmada ao citar diversos autores, entre os quais Morgan e Hunt (1994) e Doney e Cannon (1997), que enriquecem a literatura ao estudar constructos como confiança, comprometimento, interdependência, interações, valores compartilhados, balanceamento de poder, adaptação e satisfação mútua.

Morgan e Hunt (1994) tratam comprometimento e confiança como variáveis-chaves pelo fato de encorajarem os profissionais de marketing a (1) trabalhar para preservar os investimentos em relacionamento, por meio da cooperação com os parceiros de troca; (2) resistir a alternativas atraentes de curto prazo em favor de benefícios esperados para o longo prazo; e (3) visualizar ações potenciais de alto risco como prudentes, dada a crença de que os parceiros não agirão de forma oportunista.

A confiança, neste contexto, é definida como o sentimento de certeza e segurança de uma parte na integridade da outra, associada à honestidade, responsabilidade, competência, justiça e benevolência. Berry (1996, p. 42) afirma que "a natureza inerente dos serviços, aliada com a abundante falta de confiança na América, posiciona a confiança como a principal ferramenta de Marketing de Relacionamento de uma companhia” (p. 42); por conseguinte, a estabilidade de relacionamentos cooperativos é aumentada, quando a confiança é alta (GHEMAWAT, 2000). Doney e Cannon (1997) determinam que a confiança em um fornecedor é construída por meio da confiança nos vendedores e na própria empresa. Lau e Lee (1999) defendem que a confiança é a disposição em contar com as outras partes em face do risco. Brei (2001) desenvolve um modelo sobre antecedentes e conseqüência do constructo confiança, concluindo que a lealdade do cliente é positivamente influenciada pela confiança e pelo valor percebido pelo cliente.

Crosby et al. (1990) e Dwyer e Oh (1987) estudam a confiança como característica ou aspecto da qualidade do relacionamento, argumentando que as oportunidades de vendas futuras dependerão basicamente desta qualidade do relacionamento, refletida pela confiança e satisfação das partes envolvidas (LEMON et al., 2002). Diferentemente, Moorman et al. (1992) e Moorman et al. (1993) conceituam 
confiança como determinante central dos relacionamentos, indicando, por meio de pesquisa exploratória, duas linhas de pensamento sobre sua definição. A primeira a visualiza como crença, sentimento ou expectativa acerca da validade do processo de troca com o parceiro, definição com a qual grande parte da pesquisa em marketing se identifica. A segunda, por sua vez, trata a confiança como comportamento de uma parte em relação à outra, permeado pela incerteza e vulnerabilidade da parte que confia na outra. Os autores defendem que ambas as dimensões devem estar presentes para a confiança existir. Sirdeshmukh et al. (2002) propõem uma abordagem dinâmica de confiança, incluindo dimensões de competência operacional, benevolência e orientação para solução de problemas.

Já o comprometimento pode ser resumido como a crença dos parceiros na importância do relacionamento e de sua manutenção, a ponto de garantir máximos esforços para tal. A idéia implícita é a de que os relacionamentos caracterizados por confiança são tão valorizados, que as partes desejarão comprometer-se com tais relacionamentos, deixando claro que a confiança é o principal determinante do comprometimento relacional, e justificando a relação positiva existente entre as variáveis confiança e comprometimento.

Coote e Rowe (2001), em estudo sobre os determinantes da lealdade do consumidor no setor hoteleiro, afirmam que a lealdade caminha passo a passo com a noção de comprometimento, estágio máximo de um relacionamento proposto por Dwyer et al. (1987). O comprometimento afetivo, ou componente de atitude do comprometimento (ANDERSON; WEITZ, 1989), é definido por Scanzoni (1979) como o desejo duradouro de manter o relacionamento com a outra parte. Já o comprometimento instrumental refere-se à ação afirmativa das partes no sentido de demonstrar real interesse no relacionamento, mais que a mera promessa (GUNDLACH et al., 1995). A terceira dimensão do comprometimento, denominada temporal, refere-se às expectativas de continuidade do relacionamento (HEIDE; JOHN, 1990; SCANZONI, 1979).

Para o presente estudo, utilizou-se a escala para mensuração de Marketing de Relacionamento proposta por Wilson e Vlosky (1997), composta de um conjunto de seis variáveis latentes, que desempenham papel determinante na diferenciação entre os relacionamentos tradicionais e os de parceria. Estas variáveis latentes são: (1) dependência; (2) nível de comparação de alternativas; (3) investimentos específicos no relacionamento; (4) partilha de informações; (5) confiança e (6) comprometimento.

\section{Hipóteses de Pesquisa}

Tendo como base a revisão da literatura sobre serviços ao cliente e Marketing de Relacionamento, desenvolvem-se dez hipóteses de pesquisa. 
Nível de Oferta de Serviços ao Cliente e Investimentos no Relacionamento Comercial. Os processos de troca naturalmente reconhecidos e apreciados são aqueles com maior valor percebido pelo cliente. Quinn et al. (1998) afirmam que, com a queda dos custos de produção, o valor das trocas passa a ter origem em atributos que apenas os serviços podem criar. Lovelock e Wright (2001) afirmam que, quanto mais valioso o serviço, maiores serão os recursos e esforços que as pessoas ou organizações estarão dispostos a empregar para obtê-lo. As organizações com predisposição comportamental para desenvolver relacionamentos tenderão a investir de forma mais marcante em processos de trocas relacionais com parceiros diferenciados. Assim, a primeira hipótese de pesquisa estabelece:

H1: Quanto maior a oferta de serviços ao cliente do pólo fornecedor, maiores serão os investimentos do pólo comprador no relacionamento comercial.

Nível de Oferta de Serviços ao Cliente e Dependência. Entre os indicadores da variável latente dependência, propostos por Wilson e Vlosky (1997) e adaptados para o contexto de serviços, ilustra-se a percepção do cliente de que seria difícil substituir o atual fornecedor sem causar impactos que pudessem ser originados do decréscimo do valor de uma transação, remetendo ao conceito de oferta de serviços ao cliente como fonte geradora de valor agregado (BERRY; PARASURAMAN, 1992; INNIS; LA LONDE, 1994; LOVELOCK; WRIGHT, 2001; ZEITHAML, 1982; QUINN et al., 1998). Heide e John (1988) apontam algumas possibilidades de aumento da dependência, estando todas relacionadas ao impacto da diferenciação da oferta de parceiros nos resultados da empresa, tais como quando (1) os resultados obtidos na relação são importantes e altamente valiosos, sendo o volume da troca alta; (2) os resultados obtidos excedem resultados que estariam disponíveis para ele na melhor alternativa de vendedor; e (3) quando existem poucas alternativas ou potenciais recursos de troca. Neste sentido, a segunda hipótese assume:

H2: Quanto maior a oferta de serviços ao cliente do pólo fornecedor, maior o sentimento de dependência do pólo comprador em relação ao fornecedor.

Nível de Oferta de Serviços ao Cliente e Nível de Comparação de Alternativas. Os serviços ao cliente aumentam o valor ou diferenciam os serviços daqueles criados pelos concorrentes (GRÖNROOS, 1995). Bowen et al. (1989) definem os serviços ao cliente como fonte potencial de diferenciação do produto, conferindo à empresa uma vantagem competitiva em relação aos seus competidores e, por conseguinte, tornando-a destacada na comparação de alternativas. Deste modo, a terceira hipótese de estudo define: 
H3: Quanto maior a oferta de serviços ao cliente do pólo fornecedor, mais vantajosa será a comparação de alternativas realizada pelo pólo comprador.

Nível de Oferta de Serviços ao Cliente e Confiança. Enquanto oferta extra, e ampliada, os serviços ao cliente surgem como fonte de provimento de valores adicionais à transação (BERRY; PARASURAMAN, 1992; BOWEN et al., 1994; INNIS; LA LONDE, 1994; LOVELOCK; WRIGHT, 2001; ZEITHAML, 1982;), demonstrando o interesse da empresa fornecedora em manter o relacionamento com seus clientes por meio da geração de valor (GRÖNROOS, 2000). Como fonte de diferenciação, a oferta de serviços ao cliente torna-se sujeito ativo na manutenção e aprimoramento da qualidade do relacionamento, da qual a confiança faz parte (CROSBY et al., 1990; DWYER; $\mathrm{OH}, 1987)$. Segundo estes autores, as oportunidades de vendas futuras dependerão basicamente desta qualidade do relacionamento, refletida pela confiança e satisfação das partes envolvidas, e refinada pela oferta de serviços ao cliente. Assim, a quarta hipótese estabelece:

H4: Quanto maior a oferta de serviços ao cliente do pólo fornecedor, maior o sentimento de confiança do pólo comprador no pólo fornecedor.

Investimentos no Relacionamento e Dependência. Os investimentos feitos no relacionamento acarretam maiores custos de substituição do parceiro, tornando a manutenção do relacionamento mais importante para o alcance dos objetivos determinados (GANESAN, 1994; OWENS, 1994; WATHNE et al., 2001). Heide e John (1988), da mesma forma, expressam uma relação positiva entre a dependência e os custos de substituição de parceiros. A quinta hipótese, assim, propõe:

H5: Quanto maiores os investimentos do pólo comprador no relacionamento comercial, maior o sentimento de dependência em relação ao fornecedor.

Nível de Comparação de Alternativas e Dependência. A diferenciação positiva entre fornecedores determina seu poder de atração, podendo-se afirmar que, quanto maior for tal diferenciação entre o fornecedor atual e os demais, maior será a relação custo-benefício percebida (WILSON, 1995), e, por conseguinte, maior será o sentimento de dependência do pólo comprador em relação ao fornecedor. Heide e John (1988) apontam algumas possibilidades de aumento da dependência da empresa cliente, como quando os resultados obtidos excedem resultados que estariam disponíveis para ele na melhor alternativa de vendedor. Deste modo, a sexta hipótese sustenta: 
H6: Quanto mais vantajoso o fornecedor atual for para o pólo comprador, maior o sentimento de depedência do pólo comprador em relação ao pólo fornecedor.

Nível de Comparação de Alternativas e Confiança. A diferenciação, por sua vez, atua de forma decisiva no aprimoramento da qualidade do relacionamento, que tem como consequiência a confiança entre as partes (CROSBY et al., 1990; DWYER; OH, 1987). O nível de comparação do atual parceiro com os demais disponíveis no mercado tende a ser positivo para aqueles que proverem maior valor agregado às transações, esforço com reflexos na qualidade dos serviços e, por conseguinte, na confiança desenvolvida entre as partes. A sétima hipótese de pesquisa, assim, estabelece:

H7: Quanto mais vantajoso for o fornecedor atual para o pólo comprador, maior será o sentimento de confiança do pólo comprador no pólo fornecedor.

Troca de Informações e Confiança. O comportamento das partes, no que diz respeito à circulação de informações, é indicador-chave para a avaliação da vitalidade da interação dos parceiros (MORGAN; HUNT, 1994; MOHR; SPEKMAN, 1994). Bowersox (1998, p. 338) afirma que "o compartilhamento da informação é o aglutinador que mantém as associações (de alianças estratégicas) integradas”. Anderson e Narus (1990) e Lewin e Johnston (1997) sugerem que a comunicação acarreta o aumento da confiança entre as partes envolvidas no relacionamento, fornecendo suporte teórico à oitava hipótese de pesquisa:

H8: Quanto mais intensa a troca de informações entre o pólo comprador e o fornecedor, maior o sentimento de confiança do pólo comprador no fornecedor.

Dependência e Comprometimento. A maior dependência das partes em uma relação comercial justifica um maior interesse na sustentação da relação no longo prazo (GANESAN, 1994; GUNDLACH; CADOTTE, 1994). Os esforços e recursos dedicados à manutenção do relacionamento partem do pressuposto de que se trata de uma relação comercial considerada importante quanto aos seus produtos ou outputs (FONTENOT et al., 1998), justificando o interesse na preservação do relacionamento (GUNDLACH et al., 1995). Assim, a nona hipótese de pesquisa propõe:

H9: Quanto maior a dependência do pólo comprador em relação ao 
fornecedor, maior o comprometimento do pólo comprador com o relacionamento comercial.

Confiança e Comprometimento. Morgan e Hunt (1994) afirmam que os relacionamentos caracterizados pela confiança são tão valorizados, que as empresas desejarão comprometer-se com eles, deixando claro que a confiança é o principal determinante do comprometimento no relacionamento. Ganesan (1994) trata a confiança como um dos fatores que definem a orientação da transação no longo prazo, ao lado da interdependência. Para o autor, níveis altos de confiança, característica das trocas relacionais, habilitam as partes a focar os benefícios de longo prazo de um relacionamento, aumentando a competitividade e reduzindo os custos transacionais (NOORDEWIER et al., 1990). Com isto, a décima hipótese do estudo estabelece:

H10: Quanto maior a confiança do pólo comprador no pólo fornecedor, maior o comprometimento do pólo comprador com o relacionamento comercial.

A Figura 1 apresenta de forma gráfica o modelo representado pelas dez hipóteses de pesquisa propostas e baseadas na literatura.

Figura 1: Modelo sobre Serviços ao Cliente e Marketing de Relacionamento

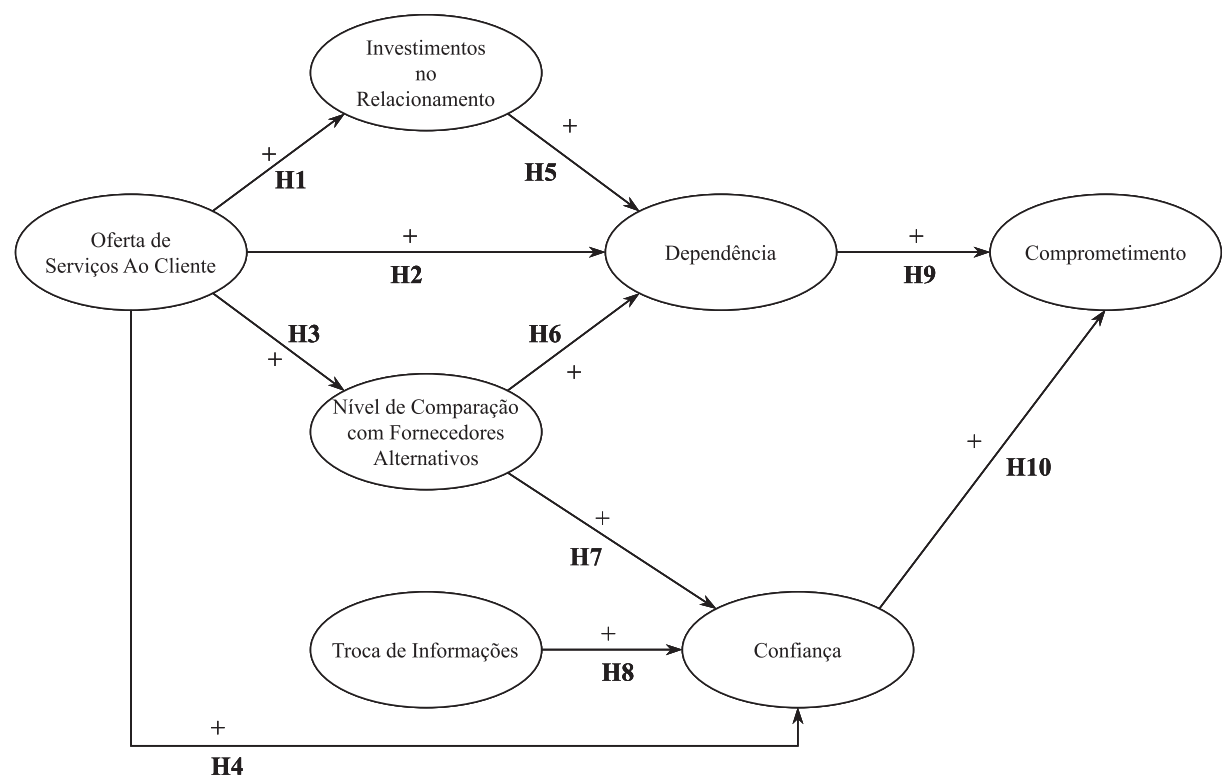




\section{Método}

\section{Amostra e Coleta de Dados}

Os dados foram coletados para avaliar a perspectiva do pólo comprador, ou seja, o ponto de vista do cliente organizacional no relacionamento com as redes hoteleiras. A população do estudo foi constituída por todos os clientes organizacionais cadastrados nas duas redes hoteleiras pesquisadas (Plaza São Rafael e Ritter Hotéis), totalizando 7.725 empresas, dos mais variados ramos e portes.

A amostra de pesquisa foi definida em 500 casos divididos entre Plaza São Rafael e Ritter Hotéis, resultando em 250 casos de clientes organizacionais por rede hoteleira. Os respondentes nos clientes organizacionais foram os responsáveis pela interação com as redes hoteleiras. O número de casos selecionados seguiu sugestão de Kline (1998), que aponta para um tamanho de amostra de, no mínimo, 250 casos por grupo. A lista de clientes foi sorteada por meio do critério de amostragem aleatória simples, tendo como único critério de seleção constar no cadastro de clientes ativos do hotel nos 12 meses anteriores. Por tratar-se de clientes organizacionais de diferentes portes e localidades, o perfil, bem como o interesse em serviços hoteleiros por parte das organizações, é bastante diverso.

O método de coleta de dados adotado foi o telefônico, em pesquisa tipo survey em corte transversal. A coleta ocorreu nos meses de setembro e outubro de 2001, período em que 500 empresas foram contatadas e 355 responderam à pesquisa, resultando um aproveitamento de $71 \%$ de casos válidos.

\section{Escalas de Mensuração}

A definição das escalas de mensuração do grau de Marketing de Relacionamento e oferta de serviços ao cliente foram objeto da etapa exploratória do estudo. A revisão da literatura pertinente aos tópicos em análise teve como propósitos propiciar suporte teórico às hipóteses de pesquisa, bem como, juntamente com entrevistas de profundidade realizadas com as duas redes hoteleiras e dez clientes organizacionais destas, auxiliar na seleção, geração e adaptação das escalas.

A escolha da escala de Wilson e Vlosky (1997) para mensuração do grau de Marketing de Relacionamento ocorreu nesta etapa do estudo. A tradução reversa desta escala já havia sido feita por Viana (1999), sendo adaptada para o contexto de prestação de serviços com base nas entrevistas de profundidade, anteriormente mencionadas. Este procedimento de adaptação da escala foi sugerido por um dos 
autores da escala, Dr. Richard Vlosky, em consulta por e-mail. Três indicadores da escala de Wilson e Vlosky (1997) não apresentaram coerência com o setor de prestação de serviços e, por esta razão, foram eliminados da escala, sendo eles as variáveis $\mathrm{V}^{(1)}$ e $\mathrm{V} 4^{(2)}$ do constructo Dependência e a variável V22 ${ }^{(3)}$ do constructo Confiança.

Os indicadores da oferta de serviços ao cliente foram gerados nas mesmas entrevistas de profundidade, tendo como ponto de partida os itens propostos por Slongo (1994), baseados em La Londe e Zinszer (1976), além de outros indicadores que surgiram nos contatos de entrevista.

As duas escalas, tanto a de serviços ao cliente quanto a de Marketing de Relacionamento, são do tipo intervalar, com o intuito de medir a intensidade dos sentimentos dos respondentes (CHURCHILL, 2001). Inicialmente, escolheu-se a escala de cinco pontos; todavia, em razão da coleta de dados ter sido feita por telefone, a solução mais razoável constitui-se na adaptação da escala de cinco pontos para outra de seis pontos de duas etapas, as quais podem ser lidas pelo entrevistador e entendidas pelo respondente de forma mais fácil (AAKER et al., 2001).

Deste modo, o questionário foi composto por 55 variáveis diretamente observáveis dos constructos, sendo 27 de Marketing de Relacionamento e 28 de serviços ao cliente.

\section{Procedimentos Estatísticos}

De acordo com as características do estudo, utilizou-se a modelagem de equações estruturais (SEM - Structural Equation Modeling), capaz de analisar múltiplos relacionamentos de variáveis dependentes e independentes (Hair et al., 1998). Os softwares estatísticos utilizados foram SPSS for Windows 10.0 e Lisrel 8.12i.

\section{Análise dos Dados e Resultados}

\section{Estimação do Modelo de Mensuração}

A validação dos constructos teóricos foi realizada por meio do uso da análise fatorial confirmatória (AFC). A técnica de AFC dá-se por meio do teste de um modelo de mensuração das supostas relações entre os indicadores (variáveis 
manifestas) e seus constructos (variáveis latentes). A AFC teve como intuito verificar se a estrutura proposta para o modelo de mensuração é plausível, por meio de desenhos para validação de constructos e refinamento de escalas (MACCALLUM; AUSTIN, 2000).

Como resultado, dois indicadores foram eliminados da análise: o item 6 do constructo Nível de Comparação com Fornecedores Alternativos ${ }^{(4)}$ foi retirado por não ser significativo, enquanto o item 11 do constructo Investimentos no Relacionamento $^{(5)}$ foi excluído por sua carga fatorial ter sido muito baixa. Além disso, a equação que indica o constructo Serviços ao Cliente como preditor do Nível de Comparação com Fornecedores Alternativos foi excluída por não ser significativa.

Em segunda rodada de reespecificações, incluiu-se uma nova relação entre constructos de Marketing de Relacionamento no modelo inicialmente proposto. O acréscimo desta nova relação, prevendo relacionamento positivo entre Troca de Informações e Investimentos no Relacionamento, doravante denominada hipótese 11, ocorreu com o devido julgamento e fundamentação teórica da hipótese.

O modelo de mensuração final aparenta ser coerente, demonstrando que os indicadores e constructos realmente representam o que se propõe a mensurar.

\section{Tabela 1: Fidedignidade e Variância Extraída para os Constructos Latentes}

\begin{tabular}{l|l|l}
\hline Constructo & Fidedignidade & Variância Extraída \\
\hline Dependência & 0,7966 & $57,67 \%$ \\
Nível de Comparação com Fornecedores & 0,7078 & $50,61 \%$ \\
Alternativos & 0,8476 & $56,21 \%$ \\
Investimentos no Relacionamento & 0,9647 & $93,19 \%$ \\
Troca de Informações & 0,7246 & $31,01 \%$ \\
Confiança & 0,8242 & $40,46 \%$ \\
Comprometimento & 0,9477 & $40,10 \%$ \\
Oferta de Serviços ao Cliente &
\end{tabular}

Fonte: coleta de dados

Tabela 2: Coeficientes de Determinação das Equações Estruturais

\begin{tabular}{l|l}
\hline Equação & $\mathbf{R}^{\mathbf{2}}$ \\
\hline DEP = NC IR SC & 0,54 \\
IR = SC TI & 0,40 \\
CON = NC TI SC & 0,45 \\
COM = DEP COM & 0,77 \\
\hline
\end{tabular}

Fonte: output do software Lisrel 8.12i 


\section{Modelo Estrutural}

O procedimento utilizado foi o de maximum likelihood, ou máxima verossimilhança (MLE), técnica mais usual de estimação. O grau de ajustamento geral é obtido por meio do grau de correspondência entre a matriz de correlação de entrada, ou os dados observados, e as predições indicadas pelo modelo de acordo com a teoria. Dentre as várias medidas de ajustamento desenvolvidas pela Estatística, não se pode apontar uma que resuma a adequação dos dados às hipóteses estabelecidas. Hair et al. (1998) sugerem a utilização de uma combinação de vários índices, aprimorando o poder explicativo da estrutura teórica estudada. A verificação do ajuste geral do modelo foi feita utilizando-se uma série de medidas que procuram traduzir em números o grau de ajuste do modelo estimado. Existem muitas medidas de ajuste disponíveis na saída do software Lisrel, mas algumas delas são mais utilizadas devido a propriedades desejáveis, quais sejam: QuiQuadrado $\left(\chi^{2}\right)$, Goodness-of-Fit Index (GFI), Comparative Fit Index (CFI), Normal Fit Index (NFI), Nonnormed Fit Index (NNFI), e Root Mean Square Error of Approximation (RMSEA).

A análise do modelo inicialmente proposto (ver Figura 1) aponta um grau de ajustamento relativamente fraco do conjunto de constructos de Marketing de Relacionamento e serviços ao cliente em relação aos padrões consagrados pela literatura $\left(\chi^{2}=4.628,46\right.$; $\mathrm{DF}=1.419 ; \mathrm{p}<0,01$; $\mathrm{GFI}=0,73$; $\mathrm{CFI}=0,74$; $\mathrm{NFI}=0,69$; $\mathrm{NNFI}=0,76$; RMSEA $=0,080)$.

Mediante reespecificações com a análise fatorial confirmatória (eliminação de dois indicadores e uma relação de causalidade) e a inclusão de nova hipótese de pesquisa, o modelo estrutural foi reestimado. Todos os parâmetros resultaram significativos, sobretudo o parâmetro referente à inclusão da nova relação de causalidade (hipótese 11). Os índices de ajustamento permitem a aceitação inicial do modelo, prevendo associações entre oferta de serviços ao cliente e atributos indicadores de Marketing de Relacionamento.

\section{Tabela 3: Medidas de Ajuste para o Modelo de Marketing de Relacionamento e Serviços ao Cliente}

\begin{tabular}{llllllllll}
\hline Modelo & $\chi^{2}$ & DF & $\chi^{2}$ /DF & $\begin{array}{l}\text { Valor } \\
\text { de } \boldsymbol{p}\end{array}$ & GFI & CFI & NFI & NNFI & RMSEA \\
\hline Inicial & $4.628,46$ & 1419 & 3,26 & $<0,01$ & 0,73 & 0,74 & 0,69 & $\mathbf{0 , 7 6}$ & $\mathbf{0 , 0 8 0}$ \\
\hline Revisado & $4.233,80$ & 1312 & 3,23 & $<0,01$ & 0,76 & 0,80 & 0,72 & 0,78 & 0,079 \\
\hline
\end{tabular}

Fonte: output do software Lisrel $8.12 i$

Das dez hipóteses que constituem o modelo originalmente proposto, uma não pôde ser aceita em função das reespecificações realizadas para o aprimoramento 
do modelo; mas uma nova relação foi inserta em decorrência da aceitação da hipótese 11. A Tabela 4 apresenta um resumo dos resultados encontrados.

\section{Tabela 4: Resumo dos Resultados para o Modelo Revisado}

\begin{tabular}{ccccc}
\hline Hipótese & Relação de Dependência & Coeficiente & P & Avaliação \\
\hline H1 & $\begin{array}{c}\text { Nível de Oferta de Serviços ao Cliente } \rightarrow \text { Investimentos } \\
\text { no Relacionamento }\end{array}$ & 0,23 & $\mathrm{p}<0,01$ & Aceita \\
\hline H2 & Nível de Oferta de Serviços ao Cliente $\rightarrow$ Dependência & 0,14 & $\mathrm{p}<0,05$ & Aceita \\
\hline H3 & Nível de Oferta de Serviços ao Cliente $\rightarrow$ Nível de & \multicolumn{2}{c}{ Hipótese Rejeitada } \\
\hline Comparação de Alternativas & Nível de Oferta de Serviços ao Cliente $\rightarrow$ Confiança & 0,55 & $\mathrm{p}<0,01$ & Aceita \\
\hline H5 & Investimentos no Relacionamento $\rightarrow$ Dependência & 0,64 & $\mathrm{p}<0,01$ & Aceita \\
\hline H6 & Nível de Comparação de Alternativas $\rightarrow$ Dependência & $-0,12$ & $\mathrm{p}<0,05$ & Aceita \\
\hline H7 & Nível de Comparação de Alternativas $\rightarrow$ Confiança & 0,15 & $\mathrm{p}<0,05$ & Aceita \\
\hline H8 & Troca de Informações $\rightarrow$ Confiança & 0,18 & $\mathrm{p}<0,01$ & Aceita \\
\hline H9 & Dependência $\rightarrow$ Comprometimento & 0,39 & $\mathrm{p}<0,01$ & Aceita \\
\hline H10 & Confiança $\rightarrow$ Comprometimento & 0,71 & $\mathrm{p}<0,01$ & Aceita \\
\hline H11 & Troca de Informações $\rightarrow$ Investimentos no & 0,54 & $\mathrm{p}<0,01$ & Aceita \\
\hline
\end{tabular}

Em fase dos ajustes, eliminações e inserção de nova hipótese realizados sobre o modelo inicial, fundamentados basicamente na revisão da literatura pertinente a Marketing de Relacionamento e serviços ao cliente, o modelo final é apresentado na Figura 2.

\section{Figura 2: Modelo Final sobre Serviços ao Cliente e Marketing de Relacionamento}

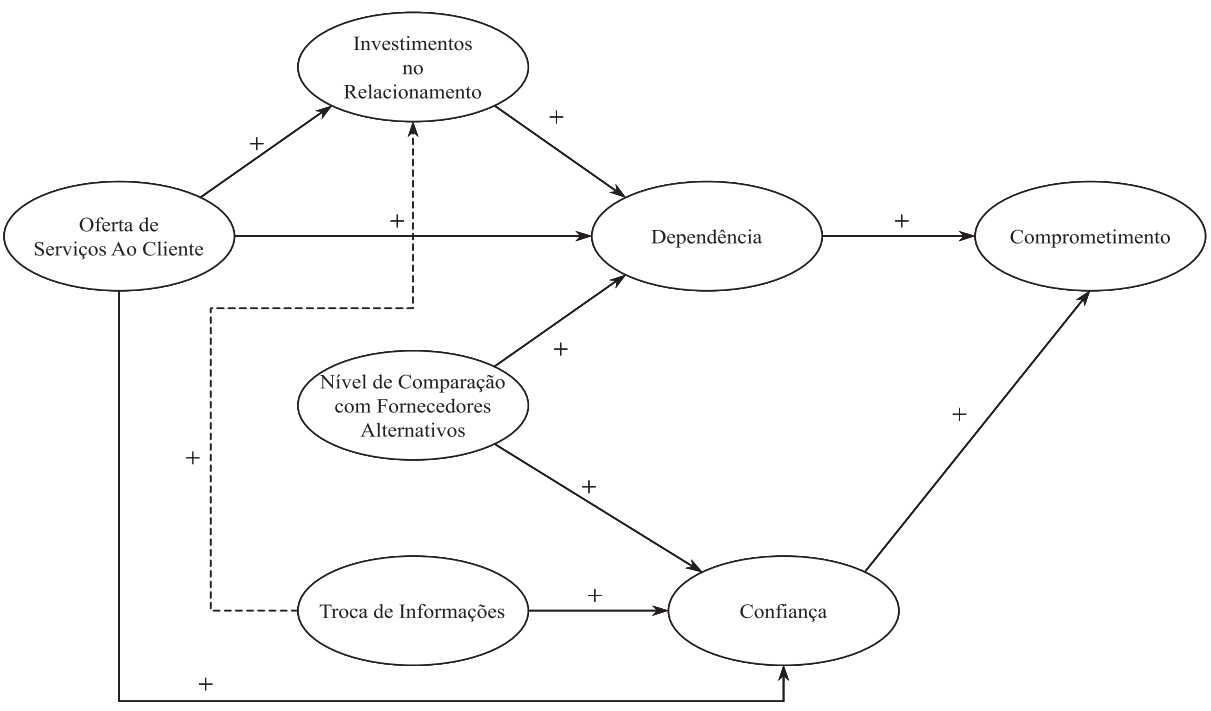

A próxima seção discute tópicos sobre os resultados obtidos na pesquisa. 


\section{Dıscussão}

A oferta de serviços ao cliente do pólo fornecedor afeta os investimentos no relacionamento e os sentimentos de dependência e confiança do pólo comprador. Assim, quanto maior a oferta de serviços ao cliente, maiores são os investimentos no relacionamento, os sentimentos de dependência e confiança do pólo comprador no relacionamento comercial.

O constructo dependência é positivamente afetado não apenas pela oferta de serviços ao cliente, mas também pelas variáveis latentes, nível de comparação com fornecedores alternativos e investimentos no relacionamento.

Além disso, a troca de informações atua como antecedente dos constructos investimentos no relacionamento e confiança. O sentimento de confiança do pólo comprador no pólo fornecedor é também afetado positivamente pelo nível de comparação com fornecedores alternativos.

Dentre os vínculos causais, destaca-se o constructo comprometimento, afetado pela dimensão dependência e altamente influenciado pela dimensão confiança. Este forte relacionamento era esperado em função da ampla e consistente literatura que sustenta a hipótese, especialmente Morgan e Hunt (1994), que posicionam confiança e comprometimento como variáveis mediadoras de Marketing de Relacionamento. Os constructos confiança e dependência atuam como antecessores do comprometimento do pólo comprador com o relacionamento comercial. Neste sentido, em especial a variável latente confiança desempenha papel determinante na manutenção e desenvolvimento de relacionamentos contínuos, duradouros e com benefícios mútuos.

\section{Conclusões}

Este trabalho revela evidências que apóiam o impacto da oferta de serviços ao cliente no conceito de Marketing de Relacionamento, analisado por meio de seis variáveis latentes propostas por Wilson e Vlosky (1997), com foco no estabelecimento de vantagens competitivas sustentáveis para as organizações envolvidas.

Os resultados desta pesquisa são de grande valia para as organizações, sugerindo como as empresas podem construir e sustentar vantagens competitivas no longo prazo por meio da oferta de serviços ao cliente e manutenção de relacionamentos duradouros e baseados em benefícios mútuos. São analisados e testados 
constructos essenciais e pertinentes à diferenciação das organizações, como a oferta de serviços ao cliente e ao desenvolvimento de relacionamentos, como a troca de informações, investimentos no relacionamento, nível de comparação com alternativas, dependência, confiança e comprometimento. $\mathrm{Na}$ alçada gerencial, a adoção de uma estratégia desta natureza tem muitas aplicações para estrategistas e negociadores.

Destaca-se como principal contribuição acadêmica e gerencial deste trabalho o desenvolvimento de uma estrutura teórica de relacionamentos causais entre variáveis latentes de Marketing de Relacionamento e oferta de serviços ao cliente. Apesar de as próprias origens do Marketing de Relacionamento estarem intimamente vinculadas à escola de serviços, no início da década de 80, poucos são os estudos brasileiros com abordagens confirmatórias sobre os efeitos das relações entre serviços ao cliente e Marketing de Relacionamento nas empresas.

As escalas para mensuração de Marketing de Relacionamento, adaptada de Wilson e Vlosky (1997), e de oferta de serviços ao cliente mostraram-se válidas e confiáveis por meio das avaliações da validade de conteúdo e dos constructos.

No que diz respeito ao modelo estrutural proposto, verificavam-se índices de ajustamento adequados em face da complexidade do modelo e do tamanho da amostra, contendo 355 casos. Nove das dez hipóteses inicialmente propostas foram estatisticamente comprovadas. Além disso, uma nova relação de causalidade foi adicionada à estrutura, devidamente fundamentada na literatura e melhorando sensivelmente os índices de ajustamento.

\section{Limitações do Estudo e Sugestões para Pesquisas Futuras}

Este trabalho tem como foco o relacionamento comercial entre duas redes hoteleiras de Porto Alegre e seus maiores clientes organizacionais, selecionados a partir de amostra não probabilística, em abordagem descritiva do tipo corte transversal. Deste modo, a primeira limitação consiste na impossibilidade de realizar generalizações para o setor hoteleiro em escopo geográfico ampliado, bem como para outros setores de atividade. Sugere-se, assim, a reaplicação deste trabalho em outros contextos de negócios, seja envolvendo produtos ou serviços, seja para o mesmo setor analisado em diferente região geográfica.

Sugerem-se, também, pesquisas que contemplem modelos rivais ou concorrentes ao proposto neste estudo para a aproximação do modelo teórico com a realidade. A pesquisa com seis variáveis latentes de Marketing de Relacionamento e uma de serviços ao cliente é passível de proposição de novas inter-relações. Assim, conforme Hair et al. (1998), existe uma variedade de modelos teóricos que podem ser testados com dados empíricos. 
Por fim, este trabalho procurou colaborar para o avanço do conhecimento em marketing, constituindo-se em mais um passo no sentido de trazer contribuições para futuras investigações sobre os temas relacionamento e serviços ao cliente. A busca deste conhecimento é infinita, e dela depende o progresso da disciplina. Por esta razão, espera-se ter estimulado novas pesquisas e debates que envolvem marketing, em especial nas áreas de Marketing de Relacionamento e oferta de serviços ao cliente, extremamente em evidência nos últimos anos em face da crescente complexidade dos mercados.

\section{Artigo recebido em 11.09.2003. Aprovado em 05.05.2004.}

\section{Notas}

\footnotetext{
${ }^{1}$ Dependência (V3) - Seria difícil para nossa empresa substituir as vendas e lucros gerados por este fornecedor.

${ }^{2}$ Dependência (V4) - Seria difícil para este fornecedor substituir as vendas e lucros gerados pela nossa empresa.

${ }^{3}$ Confiança (V22) - Nós aceitaríamos produtos deste fornecedor sem conferir a precisão das quantidades.

${ }^{4}$ Item 6 - Comparado com outros hotéis, nosso relacionamento com este hotel é melhor.

${ }^{5}$ Item 11 - Os investimentos para desenvolver um relacionamento com este hotel são facilmente transferíveis
}

\section{ReferênCias Bibliográficas}

AAKER, D.;

KUMAR, V;

DAY, G.

Marketing research. $7^{\mathrm{a}}$ ed., New York:

John Wiley and Sons, 2001.

\section{ANDERSON, J.;}

NARUS, J.

A model of distributor firm and manufacturer firm working partnerships.

Journal of Marketing, v. 54, n. 1, p. 4258, jan. 1990.
BERRY,L.L.

Relationship marketing. In: BERRY, L.L.; SHOSTACK, G. L.; UPAH, G. D. (Eds.). Emerging perspectives of services marketing, American Marketing Association, Chicago, IL, 1983. p. 2538

BERRY, L.L.;

PARASURAMAN, A.

Serviços de marketing: competindo através da qualidade. São Paulo: Maltese-Norma, 1992. 
BOWEN, D. E.;

SIEHL, C.;

SCHNEIDER, B.

A framework for analyzing customer service orientations in manufacturing. Academy of Management Review, Los Angeles, v. 14, n. 1, p.75-95, apr., 1989.

\section{BOWERSOX, D. J.}

Os benefícios estratégicos das alianças logísticas. In: MONTGOMERY, C. A.; PORTER, M. E. Estratégia: a busca da vantagem competitiva. Rio de Janeiro: Campus, 1998. cap. 5, p. 331-346.

\section{CHURCHILL, J., G.A.}

Basic marketing research. $4^{\mathrm{a}}$ ed., Orlando: Dryden Press, 2001.

CROSBY, L.A.;

EVANS, K.R.;

COWLES, D.

Relationship quality in services selling: an interpersonal influence perspective. Journal of Marketing, [S.L.], n.3, p. 6881, july 1990.

DICKSON, P. R.

Marketing management. $2^{\mathrm{a}}$ ed., Orlando: Harcourt Brace \& Company, 1997.

DONEY, P. M.;

CANNON, J. P.

An examination of the nature of trust in buyer-seller relationships. Journal of Marketing, [S.L.], n. 2, p. 35-51, apr. 1997.

DWYER, F. R.;

$\mathrm{OH}, \mathrm{S}$.

Output sector munificence effects on the internal political economy of marketing channels. Journal of Marketing Research, [S.L.], V. 53, n. 24, p. 347-358, nov. 1987.
DWYER, F. R.;

SCHURR, P.;

$\mathrm{OH}, \mathrm{S}$.

Developing buyer seller relationships. Journal of Marketing, [S.L.], v. 51 n.2, p.11-27, apr. 1987.

FONTENOT, R. et al.

Effect of buyer-seller relationship structure on firm performance. American Marketing Association Winter Educators' Conference, Austin, Texas, 1998.

\section{GANESAN, S.}

Determinants of long-term orientation in buyer-seller relationship. Journal of Marketing, [S.L.], v. 58, n.2, p. 1-19, apr. 1994.

GRÖNROOS, C.

Marketing: gerenciamento e serviços - a competição por serviços na hora da verdade. Rio de Janeiro: Campus, 1995.

Relationship marketing: the nordic school perspective. In: SHETH, J.; PARVATIYAR, A. Handbook of relationship marketing. Thousand Oaks: Sage, 2000.

GUNDLACH, G. T.;

ACHROL, R. S.;

MENTZER, J. T.

The structure of commitment in exchange. Journal of Marketing, [S.L.], v. 59, n.1, p. 78-92, jan. 1995. 
GUNDLACH, G. T.;

ACHROL, R. S.;

MENTZER, J. T.;

CADOTTE, E. R.

Exchange interdependence and interfirm interaction : research in a simulated channel setting. Journal of Marketing Research, [S.L.] v. 55, n. 31, p. 516-532, nov. 1994.

HAIR, JR., J. F. et al.

Multivariate data analysis. $5^{\mathrm{a}}$ ed., New Jersey: Prentice Hall, 1998.

HEIDE, J. B.;

JOHN, G

The role of dependence balancing in safeguarding transaction-specific assets in conventional channels. Journal of Marketing Research, [S.L.], v. 46, n. 52, p. 20-35, jan. 1988.

INNIS, D. E.;

\section{LA LONDE, B. J.}

Customer service: the key to customer satisfaction, customer loyalty and market share. Journal of Business Logistics, [S.L.], v. 15 n.1, p. 1-27, 1994.

\section{JACKSON, B.}

Winning and keeping industrial customers. Lexington: Lexington Books, 1985a.

\section{LA LONDE, B. J.;}

\section{ZINSZER, P. H.}

Customer Service: meaning and measurement. A special study report published by national council of physical distribution management, Chicago, 1976. Chicago, Irwin, 1976.

\section{LEVITT, T.}

A imaginação de marketing. São Paulo: Atlas, 1990.
LEVY,M.;

WEITZ, B. A.

Retailing management. $2^{\mathrm{a}}$ ed., Boston: Irwin, 1992.

LEWIN, J. E.; JOHNSTON, W. J.

Relationship marketing theory in practice: a case study. Journal of Business Research, [S.L.], v. 25, n. 39, p.23-31, may 1997.

\section{LOVELOCK, C.;}

WRIGHT, L.

Serviços: marketing e gestão. São

Paulo: Saraiva, 2001.

MACCALLUM, R.;

AUSTIN, J.

Applications of structural equation modeling in psychological research. Annual Review of Psychology, [S.L.], n. 51, p. 201-226, 2000.

\section{MACNEIL, I.}

The many futures of contracts. Southern California Law Review, n. 47, p. 691-816, 1974.

Contracts: adjustment of long-term economic relations under classical, neoclassical and relational contract law. Northwestern University Law Review, n. 72, p. 854-902, 1978.

MOHR, J.;

SPEKMAN, R.

Characteristics of partnership success: partnership attributes, communication behavior, and conflict resolution techniques. Strategic Management Journal, London, n.15, p. 135-152, aug. 1994. 
MORGAN, R.;

HUNT, S.

The commitment-trust theory of relationship marketing. Journal of Marketing, [S.L.], 58, n. 3, p. 20-38, july 1994.

NOORDEWIER, T. G;

JOHN, G;

NEVIN, J. R.

Performance outcomes of purchasing arrangements in industrial buyervendor relationships. Journal of Marketing, [S.L.], v. 54, n. 4, p. 80-93, oct. 1990.

\section{OWENS, J.}

The role of specific assets in effective exchange relationships: a conceptual framework. Relationship Marketing: Theory, Methods and Applications, Atlanta, GA, 1994.

\section{PARVATIYAR, A.;}

SHETH, J.

Paradigm shift in marketing theory and approach: the emergence of relationship marketing. Relationship Marketing: Theory, Methods and Applications. Research Conference, Atlanta, GA, may, 1994.

PORTER, M. E.

Vantagem Competitiva: técnicas para análise de indústrias e da concorrência. $15^{a}$ ed., Rio de Janeiro: Campus, 1986.

\section{QUINN, J.;}

DOORLEY,T.L.;

PAQUETTE, P. C.

Além de produtos: a estratégia baseada em serviços. In: MONTGOMERY, C. A.; PORTER, M. E. Estratégia: a busca da vantagem competitiva. Rio de Janeiro: Campus, 1998.
ROWE, W. G.;

BARNES, J.G.

Relationship marketing and sustained competitive advantage. Journal of Market-Focused Management, [S.L.], v. 2, n.3, P. 67-85, june, 1998.

\section{SHETH, J.;}

\section{PARVATIYAR, A.}

Handbook of relationship marketing. $2^{\mathrm{a}}$ ed., Thousand Oaks: Sage, 2000.

\section{SHOSTACK, G. L.}

The service marketing frontier. New York: John Wiley and Sons, 1978.

\section{SLONGO, L.A.}

Serviços ao cliente na indústria eletroeletrônica no Rio Grande do Sul. 1994. 269 f., Tese (Doutorado em Administração) - Programa de PósGraduação em Administração, Universidade de São Paulo, São Paulo.

\section{VIANA, D. A.}

A proposição de um modelo sobre marketing de relacionamento no contexto business-to-business: avaliação inicial na indústria metalmecânica do Rio Grande do Sul. 1999. 125 f. Dissertação (Mestrado em Administração) - Programa de PósGraduação em Administração, Universidade Federal do Rio Grande do Sul, Porto Alegre.

WATHNE, K. H.;

BIONG, H.;

HEIDE, J. B.

Choice of supplier in embedded markets: relationship and marketing program effects. Journal of Marketing, [S.L.], v. 65, n. 2, p.54-66, apr. 2001. 
WEBSTER, J., F.

Industrial marketing strategy. New York: John Wiley \& Sons, 1984.

The changing role of marketing in the corporation. Journal of Marketing, v.56, n. 4, p. 1-17, oct., 1992.

The future role of marketing in the organization. In: LEHMANN, D. R.; JOCZ, K. E. Reflections on the futures of marketing: practice and education. Cambridge, MA: MSI, 1997. cap. 3, p. 133-178.

\section{WILSON, D.}

An integrated model of buyer seller relationships. Journal of the Academy of Marketing Science, [S.L.], n. 23, p. 335-346, Fall 1995.
WILSON, E.;

VLOSKY,R.

Partnering relationship activities: building theory from case study research. Journal of Business Research, [S,L.], v. 22 n. 39, p. 59-70, may 1997.

\section{ZEITHAML, V.A.}

How consumer evaluation processes differ between goods and services. In: LOVELOCK, C. Services Marketing. $2^{\mathrm{a}}$ ed., Prentice Hall International Inc.: New Jersey, 1982

\section{ZEITHAML, V.A.;}

BITNER, M. J.

Services Marketing: integrating customer focus across the firm. $2^{\mathrm{a}} \mathrm{ed}$., Boston: Irwin McGraw-Hill, 2000. 